\title{
Rheumatoid Factor Positivity is Associated with Lower Bone Mass in Korean Male Health Examinees without Clinically Apparent Arthritis
}

\author{
Jiwon Hwang ${ }^{1}$, Joong Kyong $\mathrm{Ahn}^{2, *}$, Jaejoon Lee ${ }^{3}$, Eun-Mi Koh ${ }^{3}$, Hoon-Suk Cha ${ }^{3, *}$ \\ ${ }^{1}$ Department of Internal Medicine, National Police Hospital, ${ }^{2}$ Department of Internal Medicine, Kangbuk Samsung Hospital, Sungkyunkwan \\ University School of Medicine, ${ }^{3}$ Department of Medicine, Samsung Medical Center, Sungkyunkwan University School of Medicine, Seoul, Korea
}

\begin{abstract}
Objective. This cross-sectional study aimed to investigate the association between rheumatoid factor (RF) positivity and bone mineral density (BMD) in male Korean subjects without any history of joint disease. Methods. Of 84,344 males who had undergone a comprehensive health checkup program in 2012, 1,390 male health examinees were recruited, whose BMD and RF results were available. A RF titer $\geq 20 \mathrm{IU} / \mathrm{mL}$ was considered positive. BMD was measured at lumbar spine (L1 L4) or hip (femoral neck and total hip) by dual-energy X-ray absorptiometry. Results. The association between RF positivity and BMD was assessed by multiple linear regression analysis. The mean age was $52.7 \pm 10.9$ years (range $19 \sim 88$ years), and RF was detected in 64 subjects (4.6\%). Demographics and laboratory data were not different between RF-positive and -negative subjects except hepatitis B surface antigen (HBsAg), which was more frequently seen in RF-positive subjects (15.6\% vs. 4.3\%, $p=0.001)$. RF-positive subjects had significantly lower BMD compared to RF-negative subjects in lumbar spine but not in total hip regardless of the existence of $\mathrm{HBsAg}\left(1.17 \pm 0.16 \mathrm{~g} / \mathrm{cm}^{2}\right.$ vs. $1.10 \pm 0.18 \mathrm{~g} / \mathrm{cm}^{2}, \mathrm{p}=0.002$ in total subjects; $1.17 \pm 0.16 \mathrm{~g} / \mathrm{cm}^{2} \mathrm{vs}$. $1.10 \pm 0.18 \mathrm{~g} / \mathrm{cm}^{2}, \mathrm{p}=0.004$ in HBsAg-negative subjects). After adjusting for multiple confounders, RF positivity was negatively associated with lumbar spine $B M D(B=-0.088$ and standard error $=0.035, p=0.011)$. Conclusion. Our results show that the presence of RF could have an unfavorable impact on bone density in apparently normal males. Additional studies to elucidate the osteoimmunological mechanism of rheumatoid factor are warranted. (J Rheum Dis 2019;26:31-40)
\end{abstract}

Key Words. Rheumatoid factor, Bone density, Male, Men's health

\section{INTRODUCTION}

The close relationship between the immune and skeletal systems has been recognized, and is referred to as 'osteoimmunology' [1]. Its discovery was through observation of bone loss in chronic inflammatory diseases such as rheumatoid arthritis (RA) and has led to major advances in showing the pathophysiological mechanisms of inflammation-driven bone loss [2]. At the same time, it was proposed that age-related osteoporosis is derived from proinflammatory status of aging, which is called "inflamm-aging" [3].

In RA, periarticular and systemic bone loss is observed very early in the disease course. Radiographic bone erosions were shown during the first two years of the disease, and even as early as a few weeks into disease onset $[4,5]$. Cortical bone loss in the hand also developed very early in the disease process [6]. Osteoporosis and reduced bone mineral density (BMD) were shown in about $25 \%$ of RA patients with symptom duration less than two

\footnotetext{
Received : May 31, 2018, Revised : August 24, 2018, Accepted : September 18, 2018

Corresponding to : Hoon-Suk Cha (iD http://orcid.org/0000-0001-5391-5376

Department of Medicine, Samsung Medical Center, Sungkyunkwan University School of Medicine, 81 Irwon-ro, Gangnam-gu, Seoul 06351, Korea. E-mail : hoonsuk.cha@samsung.com Joong Kyong Ahn (iD http://orcid.org/0000-0003-3246-4435

Department of Internal Medicine, Kangbuk Samsung Hospital, Sungkyunkwang University of School of Medicine, 29 Saemunan-ro, Jongno-gu, Seoul 03181, Korea. E-mail : mdahnjk@skku.edu

*These authors contributed equally to this work.
} 
years [7]. Positive autoimmunity, including rheumatoid factor (RF) and anti-cyclic citrullinated protein antibodies (ACPA), were known to be related to these localized and systemic BMD changes $[7,8]$, which would raise questions about the interplay between autoantibodies and bone cells. Several recent investigations have recently focused on potential mechanisms by which these autoantibodies trigger bone loss. Immune complexes, consisting of autoantibodies, can bind to Fc $\gamma$ receptors (Fc $\gamma \mathrm{R}$ ) on immune cells such as monocytes and macrophages, and this induces the production of tumor necrosis factor- $\alpha$, an osteoclastogenic cytokine that stimulate osteoclast development, which has been correlated with the amount of RF [9]. There are also emerging evidences that osteoclasts and their precursors can be directly activated by autoantibodies [10,11]. Although these findings were driven from inflammatory diseases such as RA, we might infer a certain linkage between RF and bone from this perspective.

RF was the first autoantibody known to be associated with RA, and is present in approximately $70 \% \sim 80 \%$ of RA patients. It is directed against the Fc fragment of immunoglobulin (Ig)G, with the main isotype being IgM class, but IgA and IgG classes are found as well. In spite of the low specificity, RF could help the diagnosis, and predict the prognosis of RA, as RA-associated autoantibodies. It is also found nonspecifically in other inflammatory conditions such as sarcoidosis, hepatitis B and $\mathrm{C}$ infection, and tuberculosis. These false positive reactions for RF are seen in the general population with a prevalence ranging from $1 \%$ to $5 \%[12,13]$. Moreover, levels of RF increase with age, even in healthy people [14]. With the advance of osteoimmunology, most pathophysiologic concepts have been drawn from disease condition, even though bone remodeling is a dynamic process of any living bone. While higher-than-normal levels of $\mathrm{RF}$ are often seen in individuals without specific medical conditions, very few studies have investigated the influence of RF on BMD. Therefore, we aimed to analyze the association between the presence of $\mathrm{RF}$ and reduced $\mathrm{BMD}$ in health examinees with no history of joint disease.

\section{MATERIALS AND METHODS}

\section{Subjects}

The study was undertaken as a cross-sectional design, and the population comprised males from a total of 148 ,417 subjects (age range 19 $\sim 8$ years) who participated in the health checkup program between January and December 2012 in one hospital in Seoul, South Korea. Over $80 \%$ of the participants and their spouses were employees of various companies and local governmental organizations, as the South Korean Industrial Safety and Health Law requires annual or biennial health screening of employees. The remaining participants voluntarily purchased self-paid screening examinations at the health screening center. We excluded female subjects $(n=64,073)$ in order to minimalize the estrogen effect on bone in females, including the accelerated bone loss with menopausal estrogen deficiency. Out of the male subjects $(n=84,344)$, those who did not have BMD and RF test results $(n=52,158)$ were excluded. Subjects with diabetes

84,344 male examinees were assessed for eligibility

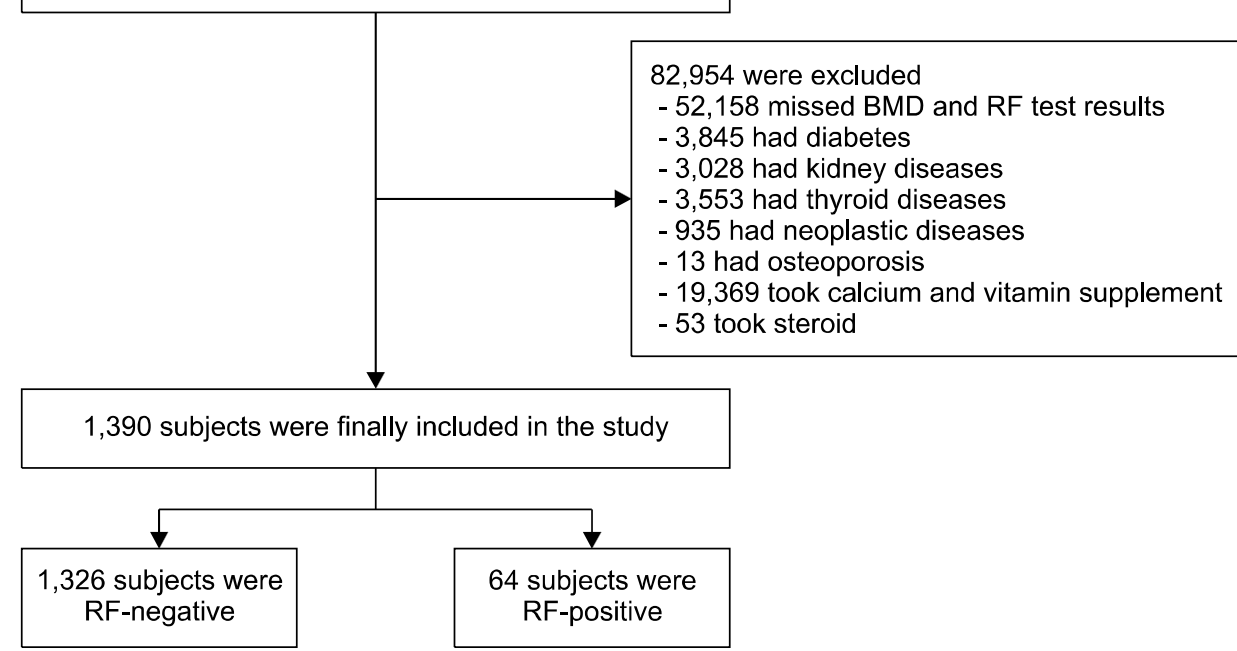

Figure 1. Selection of subjects. BMD: bone mineral density, RF: rheumatoid factor. 
$(n=3,764)$, kidney disease $(n=3,028)$, thyroid disease $(n=3,521)$, and malignancy $(n=935)$, or who were taking medicine for these conditions $(n=113)$ were excluded. Subjects treated for osteoporosis $(n=13)$, and those taking calcium and vitamin supplementation $(n=19,369)$ or steroid $(n=53)$ were also excluded. No subjects were taking disease-modifying antirheumatic drugs. This meant the analysis was performed on 1,390 male subjects with BMD and RF results available (Figure 1). Ethical approval was obtained from the Institutional Review Board of Kangbuk Samsung Hospital for patient recruitment and analysis of data (IRB no. KBSMC 2015-12-044). The Institutional Review Board exempted the requirement for informed consent for this study because a de-identified database was used to analyze data retrospectively. The study was conducted in accordance with the ethical principles of the Declaration of Helsinki.

\section{Measurements}

All subjects responded to a self-reported questionnaire from which the following information was obtained: age (years), smoking status (current smoker, ex-smoker, or never smoked) with the quantity (pack-years), alcohol consumption (grams per week), physical activity (time spent in vigorous exercise per week), medical history, and use of prescription drug and dietary supplements. Height (m) was measured to the nearest $0.1 \mathrm{~m}$ using a stadiometer with bare feet. Body weight $(\mathrm{kg})$ was measured to the nearest $0.1 \mathrm{~kg}$ on a bioimpedance analyzer (InBody 3.0 and Inbody 720; Biospace Co., Seoul, Korea) with light clothing and without shoes. Body mass index (BMI) was calculated as weight divided by height squared $\left(\mathrm{kg} / \mathrm{m}^{2}\right)$ and categorized into four groups; low ( $\leq 18.49$ $\mathrm{kg} / \mathrm{m}^{2}$ ), optimal (18.5 to $\left.24.99 \mathrm{~kg} / \mathrm{m}^{2}\right)$, overweight $(25.0$ to $\left.29.99 \mathrm{~kg} / \mathrm{m}^{2}\right)$, and obese $\left(\geq 30.0 \mathrm{~kg} / \mathrm{m}^{2}\right)$.

Blood samples were taken uniformly in the morning from the antecubital vein of participants with at least 12 hours of fasting. Serum levels of the following substances were measured as described elsewhere $[15,16]$ : glucose, aspartate aminotransferase, alanine aminotransferase (ALT), triglyceride, total cholesterol, low-density lipoprotein-cholesterol, high density lipoprotein (HDL) cholesterol, uric acid, total calcium, phosphorus, creatinine, C-reactive protein (CRP), homocysteine, hepatitis B surface antigen (HBsAg) and antibody to hepatitis $\mathrm{C}$ virus (HCV Ab). For assessing serum vitamin $\mathrm{D}$ status, total vitamin $\mathrm{D}(25-\mathrm{OH})$ was measured with a competitive immunoassay using the Elecsys vitamin D (25-OH) total assay on a Modular E170 immunoanalyzer (Roche, Basel, Switzerland). RF was measured by an immunoturbidimetric assay using a Modular P800 (Roche Diagnostics, Basel, Switzerland). RF concentration $\geq 20 \mathrm{IU} / \mathrm{mL}$ was considered as positive. Estimated glomerular filtration rate (eGFR) was calculated from age, sex, and serum creatinine using the Chronic Kidney Disease Epidemiology Collaboration (CKD-EPI) equation [17].

BMD was measured at lumbar spine (L1 L4) or hip (femoral neck and total hip) by dual-energy X-ray absorptiometry (DXA) using a GE Lunar Prodigy (Madison, WI, USA). DXA Results were expressed as grams per square centimeter $\left(\mathrm{g} / \mathrm{cm}^{2}\right)$ and T-score. For the lumbar spine $\mathrm{BMD}$, the mean BMD of four segments of the lumbar spine was used (from 1st to 4th) while for hip BMD, the total hip BMD used instead of femoral neck BMD. Osteoporosis was defined as a BMD 2.5 or more standard deviations (SD) below the sex-specific average value for young healthy adult (a T-score of $<-2.5 \mathrm{SD}$ ), according to the World Health Organization classification criteria [18]. A T-score between $-1.0 \mathrm{SD}$ and $-2.5 \mathrm{SD}$ below the young adult mean was deemed osteopenia, and a T-score $>$ -1.0 was regarded as normal. Subjects were categorized into three groups according to the T-score: normal (T-score $>-1$ ), osteopenia (T-score $\leq-1$ and $>-2.5$ ), and osteoporosis (T-score $\leq-2.5$ ).

\section{Statistical analyses}

Categorical variables were presented as numbers and percentages, and continuous variables as means $\pm S D$ or medians and interquartile ranges. The normality of distribution was assessed with the Kolmogorov-Smirnov test for all variables. Characteristics were compared between RF-positive and RF-negative groups. Differences across groups were tested using Chi-square test, Student's t-test or ANOVA tests. Correlation coefficient $r$ was measured to assess the relationship between two variables. As the relationship between RF and HBsAg positivity was significant despite of a weak correlation $(r=0.100, p=0.004$ : Supplementary Table 1), HBsAgpositive subjects were excluded in the regression analysis. To determine the independent associations of RF positivity with BMD, a multiple regression analysis was carried out, in which lumbar BMD (L1 L4) $\left(\mathrm{g} / \mathrm{cm}^{2}\right)$ served as the dependent variable and the presence of RF served as the independent variable. In the analysis, the following variables were adjusted: model 1 included age and BMI $\left(\mathrm{kg} / \mathrm{m}^{2}\right)$, model 2 additionally included alcohol 
intake (g/week), history of hypertension, and history of coronary artery disease, and model 3 further included glucose, ALT, triglyceride, HDL cholesterol, serum concentration of calcium and phosphorus, uric acid, homocysteine, ferritin, total vitamin D, and eGFR. In the multiple regression, an enter method was used, meaning that all variables were entered simultaneous into the model. For further analysis, subjects were divided into four groups by RF titer; <10.0 IU/mL, 10.0 19.99 IU/mL, $20.0 \sim 39.99 \mathrm{IU} / \mathrm{mL}$, and $\geq 40 \mathrm{IU} / \mathrm{mL}$ and the mean of the lumbar spine BMD (L1 L4) across four groups was compared using ANOVA test. All statistical analyses were performed using IBM SPSS Statistics 19.0 (IBM, Armonk, NY, USA), and a p-value less than 0.05 was considered statistically significant.

\section{RESULTS}

\section{Characteristics of study subjects}

The characteristics of the 1,390 male subject enrolled are shown in Table 1. Their mean age was $52.7 \pm 10.9$ years (range 19 $\sim 88$ years) and 64 were RF-positive (4.6\%). Smoking history was available in 1,093 subjects (78.6\%) and the proportion of current smoker was not different between the RF-negative and RF-positive groups. Drinking habits were documented in 1,235 subjects $(88.8 \%)$, and the weekly alcohol intake did not differ between the two groups. Vigorous exercise (times/week) was more frequent in the RF-negative group, but the difference was not statistical significant. Past medical history and laboratory findings were comparable between the two groups. The positivity for HBsAg was significantly higher in the RF-positive group ( $15.6 \%$ vs. $4.3 \%, \mathrm{p}=0.001$ ) but not for HCV Ab. In this regard, further analyses were conducted in both ways, including and excluding HBsAg-positive subjects for the association of $\mathrm{RF}$ with BMD. The mean level of CRP was comparable $(p=0.229)$. The presence of $R F$ had no significant relationship to aging $(r=0.045, \mathrm{p}=0.09)$.

Table 1. General characteristics by RF positivity in men $(n=1,390)$

\begin{tabular}{|c|c|c|c|}
\hline & $\begin{array}{c}\mathrm{RF}(-) \\
(\mathrm{n}=1,326)\end{array}$ & $\begin{array}{c}\mathrm{RF}(+) \\
(\mathrm{n}=64)\end{array}$ & p-value \\
\hline Age (yr) & $52.7 \pm 10.9$ & $55.0 \pm 10.7$ & 0.093 \\
\hline Body mass index $\left(\mathrm{kg} / \mathrm{m}^{2}\right)$ & $24.3 \pm 2.9$ & $24.1 \pm 2.7$ & 0.654 \\
\hline Obesity $\left(\geq 30.0 \mathrm{~kg} / \mathrm{m}^{2}\right)$ & $43(3.2)$ & $3(4.7)$ & 0.639 \\
\hline Current smoker $(n=1,093)$ & $394(37.6)$ & $20(43.5)$ & 0.665 \\
\hline Pack-years of smoking $(n=1,063)$ & $16.5 \pm 16.7$ & $17.6 \pm 15.1$ & 0.421 \\
\hline Alcohol intake (g/wk) $(\mathrm{n}=1,235)$ & $30.2 \pm 43.9$ & $27.8 \pm 41.2$ & 0.777 \\
\hline Vigorous exercise (times/wk) $(n=1,275)$ & $1.27 \pm 1.9$ & $0.88 \pm 1.6$ & 0.092 \\
\hline Hypertension & $316(23.8)$ & $9(14.1)$ & 0.098 \\
\hline Coronary artery disease & $42(3.2)$ & $4(6.2)$ & 0.158 \\
\hline Fasting glucose (mg/dL) & $98.6 \pm 14.7$ & $97.3 \pm 14.0$ & 0.487 \\
\hline Total cholesterol (mg/dL) & $200.8 \pm 35.3$ & $198.3 \pm 37.6$ & 0.580 \\
\hline High density lipoprotein cholesterol (mg/dL) & $52.9 \pm 13.9$ & $53.8 \pm 14.4$ & 0.579 \\
\hline Calcium $(\mathrm{mg} / \mathrm{dL})$ & $9.4 \pm 0.3$ & $9.4 \pm 0.4$ & 0.787 \\
\hline Phosphorus (mg/dL) & $3.4 \pm 0.4$ & $3.4 \pm 0.5$ & 0.455 \\
\hline Total vitamin $\mathrm{D}(\mathrm{mg} / \mathrm{dL})$ & $19.3 \pm 7.9$ & $21.1 \pm 9.3$ & 0.202 \\
\hline Uric acid (mg/dL) & $5.9 \pm 1.2$ & $6.0 \pm 1.3$ & 0.633 \\
\hline $\mathrm{HBsAg}(+)$ & $57(4.3)$ & $10(15.6)$ & 0.001 \\
\hline $\mathrm{HCV} \mathrm{Ab}(+)(\mathrm{n}=1,389)$ & $6(0.5)$ & 0 & 1.000 \\
\hline $\mathrm{eGFR}\left(\mathrm{mL} / \mathrm{min} / 1.73 \mathrm{~m}^{2}\right)$ & $87.5 \pm 20.0$ & $84.3 \pm 15.5$ & 0.118 \\
\hline CRP (mg/dL) & $0.06(0.02 \sim 0.12)$ & $0.08(0.02 \sim 0.19)$ & 0.229 \\
\hline RF titer (IU/mL) & $8.00(4.95 \sim 9.70)$ & $34.2(26.3 \sim 77.2)$ & $<0.001$ \\
\hline
\end{tabular}

Values are presented as mean \pm standard deviation, number of subjects with percentages or median (interquartile range). RF: rheumatoid factor, HBsAg: hepatitis B virus surface antigen, HCV Ab: antibody against hepatitis C virus, eGFR: estimated glomerular filtration rate, CRP: C-reactive protein. p-values were determined by Chi-square test for categorical variables, and Student's t-test or Mann-Whitney U-test which was for skewed continuous variables. 


\section{Distribution of BMD along the presence of RF and its levels}

Tables 2 and 3 shows the mean BMD and the categories based on T-score according to RF positivity. In the lumbar spine, the mean BMD (L1 L4) was significantly lower in the RF-positive group compared with RF-negative group $\left(1.10 \pm 0.18 \mathrm{~g} / \mathrm{cm}^{2}\right.$ vs. $\left.1.17 \pm 0.16 \mathrm{~g} / \mathrm{cm}^{2}, \mathrm{p}=0.002\right)$, and the T-score based categories also differed between the RF-positive and RF-negative groups $(\mathrm{p}<0.001)$ including HBsAg-positive subjects (Table 2). However, in the total hip measurement, neither the mean BMD nor the
T-score based categories were different between the two groups. Excluding HBsAg-positive subjects, the similar trend of BMD values was shown that a significant difference between the two groups existed only in the lumbar spine, not in the total hip (Table 3). In the lumbar spine, as the RF titer increased, which was grouped into four categories ( $<10 \mathrm{IU} / \mathrm{mL}, 10$ to $19.99 \mathrm{IU} / \mathrm{mL}, 20$ to 39.99 $\mathrm{IU} / \mathrm{mL}$, and $\geq 40 \mathrm{IU} / \mathrm{mL}$ ), the mean BMD (L1 $\sim \mathrm{L} 4)$ decreased on one-way ANOVA testing ( $\mathrm{p}=0.015$ in total subjects and $p=0.027$ in HBsAg-negative subjects, respectively) (Figure 2A and 2C). Across the four groups,

Table 2. Bone mineral density values in total subjects

\begin{tabular}{|c|c|c|c|c|c|c|c|}
\hline \multirow[b]{2}{*}{ Variable } & \multicolumn{3}{|c|}{ Lumbar spine } & \multirow[b]{2}{*}{ Variable } & \multicolumn{3}{|c|}{ Total hip } \\
\hline & $\begin{array}{c}\mathrm{RF}(-) \\
(\mathrm{n}=1,140)\end{array}$ & $\begin{array}{l}\mathrm{RF}(+) \\
(\mathrm{n}=54)\end{array}$ & p-value & & $\begin{array}{c}\mathrm{RF}(-) \\
(\mathrm{n}=249)\end{array}$ & $\begin{array}{l}\mathrm{RF}(+) \\
(\mathrm{n}=12)\end{array}$ & $p$-value \\
\hline $\begin{array}{c}\mathrm{BMD}\left(\mathrm{g} / \mathrm{cm}^{2}\right) \\
(\mathrm{n}=1,194)^{*}\end{array}$ & $1.17 \pm 0.16$ & $1.10 \pm 0.18$ & 0.002 & $\begin{array}{c}\mathrm{BMD}\left(\mathrm{g} / \mathrm{cm}^{2}\right) \\
(\mathrm{n}=261)^{*}\end{array}$ & $0.97 \pm 0.15$ & $0.94 \pm 0.23$ & 0.537 \\
\hline T-score $(n=1,194) *$ & $-0.05 \pm 1.33$ & $-0.64 \pm 1.52$ & 0.009 & T-score $(\mathrm{n}=261)^{*}$ & $0.17 \pm 1.19$ & $-0.07 \pm 1.76$ & 0.116 \\
\hline $\begin{array}{l}\text { Category based on } \\
\text { spine T-score } \\
(n=1,386)^{*}\end{array}$ & & & $<0.001$ & $\begin{array}{l}\text { Category based on } \\
\text { total hip T-score } \\
(\mathrm{n}=307)^{*}\end{array}$ & & & 0.683 \\
\hline Normal & $1,043(78.9)$ & $47(73.4)$ & & Normal & $246(83.4)$ & $9(75.0)$ & \\
\hline Osteopenia & $241(18.2)$ & $9(14.1)$ & & Osteopenia & 47 (15.9) & $3(25.0)$ & \\
\hline Osteoporosis & $38(2.9)$ & $8(12.5)$ & & Osteoporosis & $2(0.7)$ & 0 & \\
\hline
\end{tabular}

Values are presented as mean \pm standard deviation or number of subjects with percentages. RF: rheumatoid factor, HBsAg: hepatitis B surface antigen, BMD: bone mineral density. ${ }^{*}$ The discrepancy of the numbers was due to the missing values between the L1 L4 BMD results and the category, and the category was determined by the lowest T-score. p-values were determined by Chi-square test for categorical variables, and Student's t-test or Mann-Whitney U-test for skewed continuous variables.

Table 3. Bone mineral density values in subjects without $\mathrm{HBsAg*}$

\begin{tabular}{|c|c|c|c|c|c|c|c|}
\hline \multirow[b]{2}{*}{ Variable } & \multicolumn{3}{|c|}{ Lumbar spine } & \multirow[b]{2}{*}{ Variable } & \multicolumn{3}{|c|}{ Total hip } \\
\hline & $\begin{array}{c}\mathrm{RF}(-) \\
(\mathrm{n}=1,090)\end{array}$ & $\begin{array}{l}\mathrm{RF}(+) \\
(\mathrm{n}=54)\end{array}$ & p-value & & $\begin{array}{c}\mathrm{RF}(-) \\
(\mathrm{n}=240)\end{array}$ & $\begin{array}{l}\mathrm{RF}(+) \\
(\mathrm{n}=10)\end{array}$ & $p$-value \\
\hline $\begin{array}{c}\mathrm{BMD}\left(\mathrm{g} / \mathrm{cm}^{2}\right) \\
(\mathrm{n}=1,135)^{\dagger}\end{array}$ & $1.17 \pm 0.16$ & $1.10 \pm 0.18$ & 0.004 & $\begin{array}{c}\mathrm{BMD}\left(\mathrm{g} / \mathrm{cm}^{2}\right) \\
(\mathrm{n}=250)^{\dagger}\end{array}$ & $0.97 \pm 0.15$ & $0.96 \pm 0.24$ & 0.922 \\
\hline T-score $(n=1,135)^{\dagger}$ & $-0.06 \pm 1.32$ & $-0.64 \pm 1.47$ & 0.004 & T-score $(n=250)^{\dagger}$ & $0.17 \pm 1.19$ & $-0.15 \pm 1.85$ & 0.951 \\
\hline $\begin{array}{l}\text { Category based on } \\
\text { spine T-score } \\
(n=1,319)^{\dagger}\end{array}$ & & & 0.026 & $\begin{array}{l}\text { Category based on } \\
\text { total hip T-score } \\
(\mathrm{n}=262)^{\dagger}\end{array}$ & & & 0.769 \\
\hline Normal & 1,002 (79.2) & $39(72.2)$ & & Normal & $215(85.0)$ & 7 (77.8) & \\
\hline Osteopenia & 227 (17.9) & $9(16.7)$ & & Osteopenia & $36(14.2)$ & $2(22.2)$ & \\
\hline Osteoporosis & $36(2.8)$ & $6(11.1)$ & & Osteoporosis & $2(0.8)$ & 0 & \\
\hline
\end{tabular}

Values are presented asmean \pm standard deviation or number of subjects with percentages. RF: rheumatoid factor, HBsAg: hepatitis B surface antigen, BMD: bone mineral density. p-values were determined by Chi-square test for categorical variables, and Student's t-test or Mann-Whitney U-test for skewed continuous variables. ${ }^{*}$ This analysis was performed on HBsAg-negative subjects. ${ }^{\dagger}$ The discrepancy of the numbers was due to the missing values between the $L 1 \sim L 4 B M D$ results and the category, and the category was determined by the lowest T-score. 
the proportion of low bone mass of the lumbar spine increased as the RF titer increased ( $p$ for trend <0.001) (Figure 2B and 2D). On this account, further analysis for the association of RF positivity with BMD was performed only for the lumbar spine. Low bone mass (osteopenia and osteoporosis) of the lumbar spine was more prevalent in subjects aged 50 or older, compared with those younger subjects $(28.0 \%$ vs. $10.7 \%, \mathrm{p}<0.001)$, while there were no differences in the femur neck and total hip measurements. Neither the lumbar spine BMD nor the $R F$ titer showed significant correlation with age $(r=-0.022$, $\mathrm{p}=0.45$ and $\mathrm{r}=0.033, \mathrm{p}=0.22$, respectively).

\section{Association between BMD and RF positivity}

A sequential multiple regression analysis was employed to investigate the association between RF positivity and BMD (Table 4). For the first step, age and BMI were entered into the model and the RF positivity showed a significant negative association with lumbar spine BMD (model 1). For the second step, alcohol intake (g/week), history of hypertension, and history of coronary artery disease, as lifestyle factors and comorbidities, were entered, and the regression equation was still significant $(\mathrm{F}(8,814)=9.526, \mathrm{p}<0.001)$, with $\mathrm{R} 2$ of 0.084 (model 2). Lastly, laboratory values were entered simultaneously, resulting in a significant increase in $\mathrm{R} 2=0.147, \mathrm{~F}(18$, 486 ) $=4.662, \mathrm{p}<0.001$ (model 3 ), and the full model 3 demonstrated that the RF positivity could explain $14.7 \%$ of the change of the lumbar spine BMD. The RF positivity was significantly negatively correlated with the lumbar
A

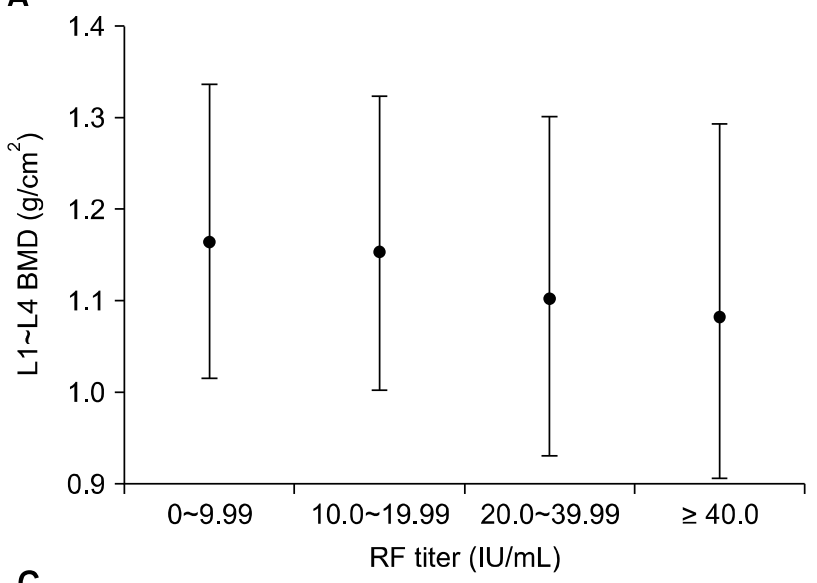

C

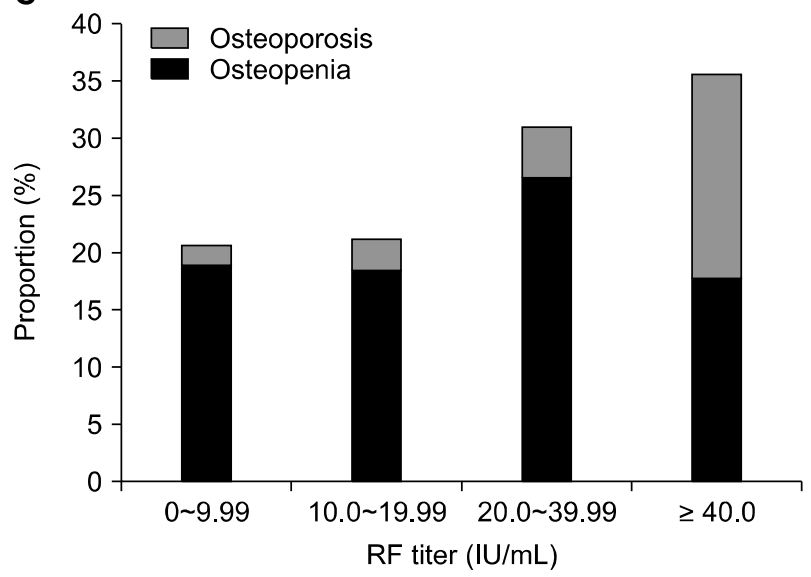

B
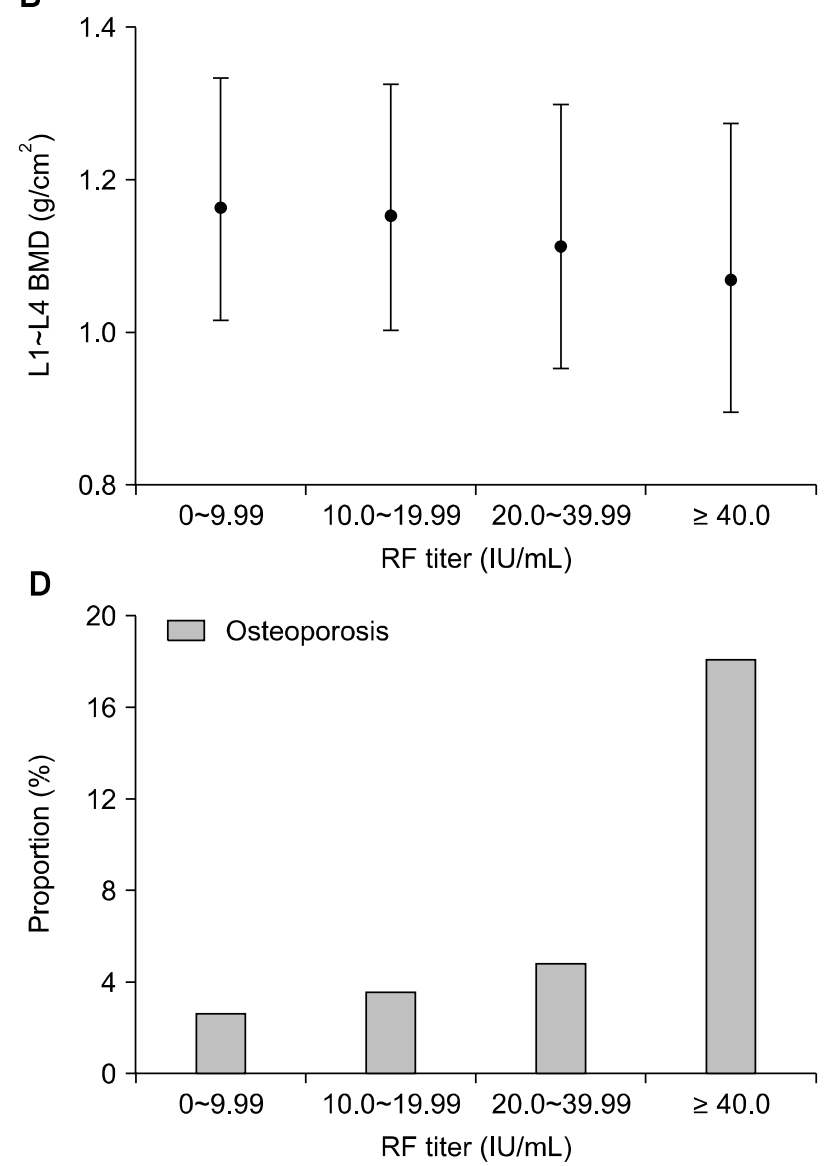

Figure 2. Mean BMD and the proportion of low bone mass in lumbar spine along RF titers. $(A)$ and $(C)$ drawn for total subjects, and (B) and (D) for HBsAg-negative subjects. (A, B) RF titers were grouped into four categories, and each rhombus dot represents the mean estimated value $( \pm 95 \% \mathrm{Cl}$ ) of lumbar $\mathrm{BMD}$ in the corresponding RF titer group. The imaginary connecting line between the dots demonstrates the decreasing tendency of BMD as the RF titers increase on one-way ANOVA testing $(p=0.015$ in $A)$ and $p=0.027$ in B, respectively). (C, D) Each column represents the percentage of subjects with osteopenia (dark gray) and osteoporosis (light gray). Across the four groups, low bone mass frequency increases as RF titers increase ( $p$ for trend $<0.001$, both). BMD: bone mineral density, RF: rheumatoid factor, HBsAg: hepatitis B surface antigen, $\mathrm{Cl}$ : confidence intervals. 
Table 4. Multiple linear regression analysis of the association between the RF positivity and lumbar spine BMD*

\begin{tabular}{ccccc}
\hline \hline BMD $\left(\mathrm{g} / \mathrm{cm}^{2}\right)$ & B & SE & p-value & $\mathrm{R}^{2}$ \\
\hline Model 1 & -0.065 & 0.023 & 0.005 & 0.101 \\
Model 2 & -0.069 & 0.028 & 0.014 & 0.084 \\
Model 3 & -0.088 & 0.035 & 0.011 & 0.147 \\
\hline
\end{tabular}

Model 1: adjusted for age, body mass index. Model 2: as for Model 1 plus: alcohol intake (g/week), smoking (pack-years), history of hypertension, and history of coronary artery disease. Model 3: as for Model 2 plus: fasting glucose, alanine aminotransferase, estimated glomerular filtration rate, uric acid, triglyceride, high density lipoprotein, homocysteine, ferritin, total vitamin $D$, and serum concentration of calcium and phosphorus. RF: rheumatoid factor, BMD: bone mineral density, B: unstandardized coefficient means the degree of change in lumbar spine BMD values $\left(\mathrm{g} / \mathrm{cm}^{2}\right)$ along the presence of RF, SE: standard error, R2: coefficient of multiple determination. ${ }^{*}$ This analysis was performed on $\mathrm{HBsAg-negative}$ subjects.

spine BMD throughout the sequential models. Other than the RF positivity, BMI had a consistent positive association with lumbar spine $B M D(B=0.017, p<0.001$ in model $1 ; \mathrm{B}=0.015, \mathrm{p}<0.001$ in model 2 ; and $\mathrm{B}=0.015, \mathrm{p}<$ 0.001 in model 3).

\section{DISCUSSION}

The significance of RF positivity with respect to bone health in subjects without arthritis has hardly been investigated. In this cross-sectional analysis of 1,390 male health examinees, RF-positive subjects had significantly lower BMD of the lumbar spine, and the presence of RF was associated with lower BMD values in the lumbar spine after adjustment of potential confounding variables. This data provides epidemiological information that indicates RF positivity may have deleterious effects on bone metabolism in males, and might be clinically useful connotations in screening male osteoporosis in the general population.

$\mathrm{RF}$ is one type of RA-associated autoantibodies, which links to the Fc portion of IgG and forms immune complexes (ICs), contributing the autoimmune inflammatory response [19]. False positive reactions are found in $1 \%$ to $5 \%$ of the general population [20]. Some studies reported a higher incidence of RF seropositivity in elderly subjects [21]. Unlike these previous reports of a higher prevalence of RF with age, our results showed no difference in the mean age between RF-positive and RF-negative subjects.
The reason for this seems to be that only $4.4 \%$ of the study population was over age 70 years, and most were below age 60 years, whereas in the other studies the RF seropositivity was predominant in age 70 years or older [21]. Moreover, in our study, age had no significant correlation either with the BMD or the RF titer. Multiple regression analyses showed a significant negative association between the presence of RF and lumbar spine BMD. Taken together, this study demonstrates that bone health in male subjects may be affected by the RF, rather than by age.

In subjects with $\mathrm{HBsAg}$, the rate of RF positivity is estimated to be $12 \% \sim 20 \%$, which is significantly higher than those without HBsAg [22-24]. A previous study suggested that HBV infection status should be considered when interpreting RF-positivity because of a strong association with RF positivity [13]. The present study also demonstrated that the positivity for HBsAg was significantly higher in the RF-positive group, and a meaningful correlation was shown between RF and HBsAg positivity in correlation analysis. Furthermore, HBsAg seropositivity has been proposed to be a risk factor for lower BMD in male [25]. On that account, the present study conducted another set of analyses, excluding HBsAg-positive subjects, which still indicated a significant association of RF positivity with BMD. This result could strengthen the genuine impact of RF on BMD, regardless of HBsAg seropositivity.

Direct evidence supporting RF as an independent risk factor for lower BMD in the general population is scarce. Going back to the 1970s and 1980s, the effect on bone has been shown mainly in patients with RA, with bone erosions more common and extensive in RF-positive patients [26,27]. After the discovery of ACPA, however, ACPA has been spotlighted rather than RF, because of the direct effect on bone by inducing osteoclast differentiation [11]. The RF ICs could also strongly enhance osteoclast differentiation via involving in their maturation process [10]. In autoimmune diseases, it is frequently observed that antibody production and IC formation are related to bone loss despite of insufficient understanding how ICs directly regulate bone metabolism [28].

In osteoclastogenesis, the receptor activator of NF-kB ligand (RANKL) is one of the key cytokines, along with macrophage colony-stimulating factor (M-CSF), and the activation of RANK also needs co-stimulatory signals including Fc receptor common $\gamma$ subunit [29]. This $\gamma$ sub- 
unit is important for receptor signaling as it is the accessory side chains of the $\mathrm{Fc} \gamma \mathrm{R}$, which recognizes IgG. There are also activating and inhibitory Fc $\gamma$ Rs with different affinities to the subclasses of IgG, and each subtype of IgGs binds the Fc $\gamma$ Rs with a certain activating-to-inhibitory ratio [30]. Under pathologic condition such as autoimmune diseases related to hypergammaglobulinemia, IgG ICs induce osteoclastogenesis by responding to the over-expressed activating Fc $\gamma$ Rs, without the counter effect by inhibitory receptors, while the activating Fc $\gamma \mathrm{R}$ signaling is counterbalanced by the inhibitory receptors in physiologic conditions [28]. In this context, we suggest that the RF ICs could act via Fc $\gamma$ Rs along their affinities to IgG in those who do not have a condition related to RF, such as our study population. A recent investigation demonstrated that RFs derived from RA patients had similar properties as those derived from healthy donors in the structure and function, and there was no difference in mutation frequency [31]. This would strengthen the suggestion for the role for RF ICs in bone resorption not only in inflammatory diseases but in general.

The multiple regression analyses did not demonstrate a meaningful association between age and lumbar spine BMD. Even though the age at which bone loss starts in males is unknown, there is some evidence that the bone remodeling rate remains low in midlife, and the loss of trabecular continuity occurs later compared with females [32]. Current clinical practice guidelines recommend screening for osteoporosis in males over the age of 70 years [33]. As the study population was mostly under the age of 60 years (72.7\%), the contribution of aging to lowering of BMD may not be adequately reflected in the present study. However, the association of BMI with the lumbar spine BMD was constant in a positive direction through models 1, 2, and 3. It is known that fat mass, and its regional distribution, can also affect BMD, although it is still controversial whether fat mass or lean mass has a greater influence on BMD. It has been observed in several studies that fat mass increased after age 74 years, and percentage fat mass increased throughout the lifetime of males [34]. The BMIs of study subjects were mostly optimal (59.4\%) or overweight (35.5\%), and only 72 subjects $(5.2 \%)$ were in the extreme ranges (low or obese BMI). In this respect, the result is consistent with previous studies, that increased BMI was protective for BMD [35].

To our knowledge, this is the first study showing a negative association between RF positivity and BMD in healthy subjects. Nonetheless, potential limitations of the present study stem from its cross-sectional design and the questionnaire-based collection of medical history. The previous fracture history of the subjects, history of osteoporosis in first-degree relatives, and the hormonal status for assessing hypogonadism were all unavailable. The results only showed an association between RF positivity and lumbar spine BMD, but as total hip BMD was assessed in just 261 subjects, this small number may restrict further analyses. In addition, the study was limited to Korean males, so caution is required in extrapolating our data to other ethnic groups and females. Further, larger longitudinal follow-up studies including measuring the BMD at other sites would provide more explicit information to verify the causal relationship between RF and BMD in the general population.

\section{CONCLUSION}

In conclusion, our study suggests that RF positivity in apparently healthy Korean male subjects was significantly associated with increased risk of lowered lumbar spine BMD assessed by DXA. This data could have practical application in screening high risk males given the epidemiologic findings that men have higher mortality and morbidity related to osteoporosis, despite a lower incidence of low bone mass compared to females. The osteoimmunological mechanisms by which RF plays a role in bone remodeling of subjects without inflammation still warrants further study.

\section{ACKNOWLEDGMENTS}

We thank Mi-Yeon Lee for her excellent statistical assistance and Bryan Lee for his making and editing on scientific figures.

\section{CONFLICT OF INTEREST}

No potential conflict of interest relevant to this article was reported.

\section{SUPPLEMENTARY DATA}

Supplementary data can be found with this article online at https://doi.org/10.4078/jrd.2019.26.1.31. 


\section{REFERENCES}

1. Pietschmann P, Mechtcheriakova D, Meshcheryakova A, Föger-Samwald U, Ellinger I. Immunology of osteoporosis: a mini-review. Gerontology 2016;62:128-37.

2. Takayanagi H. Osteoimmunology: shared mechanisms and crosstalk between the immune and bone systems. Nat Rev Immunol 2007;7:292-304.

3. Franceschi C, Bonafè M, Valensin S, Olivieri F, De Luca M, Ottaviani E, et al. Inflamm-aging. An evolutionary perspective on immunosenescence. Ann N Y Acad Sci 2000;908:244-54.

4. van der Heijde DM. Joint erosions and patients with early rheumatoid arthritis. Br J Rheumatol 1995;34 Suppl 2:74-8.

5. Machold KP, Stamm TA, Nell VP, Pflugbeil S, Aletaha D, Steiner G, et al. Very recent onset rheumatoid arthritis: clinical and serological patient characteristics associated with radiographic progression over the first years of disease. Rheumatology (Oxford) 2007;46:342-9.

6. Pye SR, Adams JE, Ward KA, Bunn DK, Symmons DP, O'Neill TW. Disease activity and severity in early inflammatory arthritis predict hand cortical bone loss. Rheumatology (Oxford) 2010;49:1943-8.

7. Güler-Yüksel M, Bijsterbosch J, Goekoop-Ruiterman YP, de Vries-Bouwstra JK, Ronday HK, Peeters AJ, et al. Bone mineral density in patients with recently diagnosed, active rheumatoid arthritis. Ann Rheum Dis 2007;66:1508-12.

8. Bugatti S, Bogliolo L, Vitolo B, Manzo A, Montecucco C, Caporali R. Anti-citrullinated protein antibodies and high levels of rheumatoid factor are associated with systemic bone loss in patients with early untreated rheumatoid arthritis. Arthritis Res Ther 2016;18:226.

9. Mathsson L, Lampa J, Mullazehi M, Rönnelid J. Immune complexes from rheumatoid arthritis synovial fluid induce FcgammaRIIa dependent and rheumatoid factor correlated production of tumour necrosis factor-alpha by peripheral blood mononuclear cells. Arthritis Res Ther 2006;8:R64.

10. Seeling M, Hillenhoff U, David JP, Schett G, Tuckermann J, Lux A, et al. Inflammatory monocytes and Fc $\gamma$ receptor IV on osteoclasts are critical for bone destruction during inflammatory arthritis in mice. Proc Natl Acad Sci U S A 2013;110:10729-34.

11. Harre U, Georgess D, Bang H, Bozec A, Axmann R, Ossipova E, et al. Induction of osteoclastogenesis and bone loss by human autoantibodies against citrullinated vimentin. J Clin Invest 2012;122:1791-802.

12. Johnson PM, Faulk WP. Rheumatoid factor: its nature, specificity, and production in rheumatoid arthritis. Clin Immunol Immunopathol 1976;6:414-30.

13. Shim CN, Hwang JW, Lee J, Koh EM, Cha HS, Ahn JK. Prevalence of rheumatoid factor and parameters associated with rheumatoid factor positivity in Korean health screening subjects and subjects with hepatitis B surface antigen. Mod Rheumatol 2012;22:885-91.

14. Shin YS, Choi JH, Nahm DH, Park HS, Cho JH, Suh CH. Rheumatoid factor is a marker of disease severity in Korean rheumatoid arthritis. Yonsei Med J 2005;46:464-70.

15. Chang Y, Kim BK, Yun KE, Cho J, Zhang Y, Rampal S, et al. Metabolically-healthy obesity and coronary artery calcification. J Am Coll Cardiol 2014;63:2679-86.
16. Zhang Y, Chang Y, Ryu S, Cho J, Lee WY, Rhee EJ, et al. Thyroid hormones and mortality risk in euthyroid individuals: the Kangbuk Samsung health study. J Clin Endocrinol Metab 2014;99:2467-76.

17. Levey AS, Stevens LA, Schmid CH, Zhang YL, Castro AF 3rd, Feldman HI, et al. A new equation to estimate glomerular filtration rate. Ann Intern Med 2009;150:604-12.

18. Kanis JA, Melton LJ 3rd, Christiansen C, Johnston CC, Khaltaev N. The diagnosis of osteoporosis. J Bone Miner Res 1994;9:1137-41.

19. Soltys AI, Axford JS, Sutton BJ. Rheumatoid factors: where are we now? Ann Rheum Dis 1997;56:285-6.

20. Hwang J, Song JU, Ahn JK. Decline of pulmonary function is associated with the presence of rheumatoid factor in Korean health screening subjects without clinically apparent lung disease: a cross-sectional study. Medicine (Baltimore) 2016;95:e3668.

21. Litwin SD, Singer JM. Studies of the incidence and significance of anti-gamma globulin factors in the aging. Arthritis Rheum 1965;8:538-50.

22. Watanabe K, Ohkubo Y, Funahashi Y, Nishimaki T, Moritoh T, Kasukawa R, et al. An investigation on rheumatoid factor of different immunoglobulin classes in hepatitis B virus carriers. Clin Rheumatol 1991;10:31-7.

23. Lee SI, Yoo WH, Yun HJ, Kim DS, Lee HS, Choi SI, et al. Absence of antibody to cyclic citrullinated peptide in sera of non-arthritic patients with chronic hepatitis B virus infection. Clin Rheumatol 2007;26:1079-82.

24. Cacoub P, Saadoun D, Bourlière M, Khiri H, Martineau A, Benhamou Y, et al. Hepatitis B virus genotypes and extrahepatic manifestations. J Hepatol 2005;43:764-70.

25. Baeg MK, Yoon SK, Ko SH, Han KD, Choi HJ, Bae SH, et al. Males seropositive for hepatitis $\mathrm{B}$ surface antigen are at risk of lower bone mineral density: the 2008-2010 Korea National Health and Nutrition Examination Surveys. Hepatol Int 2016;10:470-7.

26. el-Khoury GY, Larson RK, Kathol MH, Berbaum KS, Furst DE. Seronegative and seropositive rheumatoid arthritis: radiographic differences. Radiology 1988;168:517-20.

27. Masi AT, Maldonado-Cocco JA, Kaplan SB, Feigenbaum SL, Chandler RW. Prospective study of the early course of rheumatoid arthritis in young adults: comparison of patients with and without rheumatoid factor positivity at entry and identification of variables correlating with outcome. Semin Arthritis Rheum 1976;4:299-326.

28. Negishi-Koga T, Gober HJ, Sumiya E, Komatsu N, Okamoto $\mathrm{K}$, Sawa $\mathrm{S}$, et al. Immune complexes regulate bone metabolism through FcR $\gamma$ signalling. Nat Commun 2015;6:6637.

29. Mócsai A, Humphrey MB, Van Ziffle JA, Hu Y, Burghardt A, Spusta SC, et al. The immunomodulatory adapter proteins DAP12 and Fc receptor gamma-chain (FcRgamma) regulate development of functional osteoclasts through the Syk tyrosine kinase. Proc Natl Acad Sci U S A 2004;101:6158-63.

30. Boruchov AM, Heller G, Veri MC, Bonvini E, Ravetch JV, Young JW. Activating and inhibitory IgG Fc receptors on human DCs mediate opposing functions. J Clin Invest 2005;115:2914-23.

31. Falkenburg WJJ, von Richthofen HJ, Rispens T. On the origin of rheumatoid factors: insights from analyses of variable region sequences. Semin Arthritis Rheum 2018 Jun 24 [Epub]. DOI: 10.1016/j.semarthrit.2018.06.006. 
32. Seeman E, Bianchi G, Adami S, Kanis J, Khosla S, Orwoll E. Osteoporosis in men--consensus is premature. Calcif Tissue Int 2004;75:120-2.

33. Qaseem A, Snow V, Shekelle P, Hopkins R Jr, Forciea MA, Owens DK. Screening for osteoporosis in men: a clinical practice guideline from the American College of Physicians. Ann Intern Med 2008;148:680-4.

34. Rissanen A, Heliövaara M, Aromaa A. Overweight and an- thropometric changes in adulthood: a prospective study of 17,000 Finns. Int J Obes 1988;12:391-401.

35. Jiang $Y$, Zhang Y, Jin M, Gu Z, Pei Y, Meng P. Aged-related changes in body composition and association between body composition with bone mass density by body mass index in Chinese Han men over 50-year-old. PLoS One 2015;10: e0130400. 
pISSN: 2093-940X, elSSN: 2233-4718

Journal of Rheumatic Diseases Vol. 26, No. 1, January, 2019

https://doi.org/10.4078/jrd.2019.26.1.31

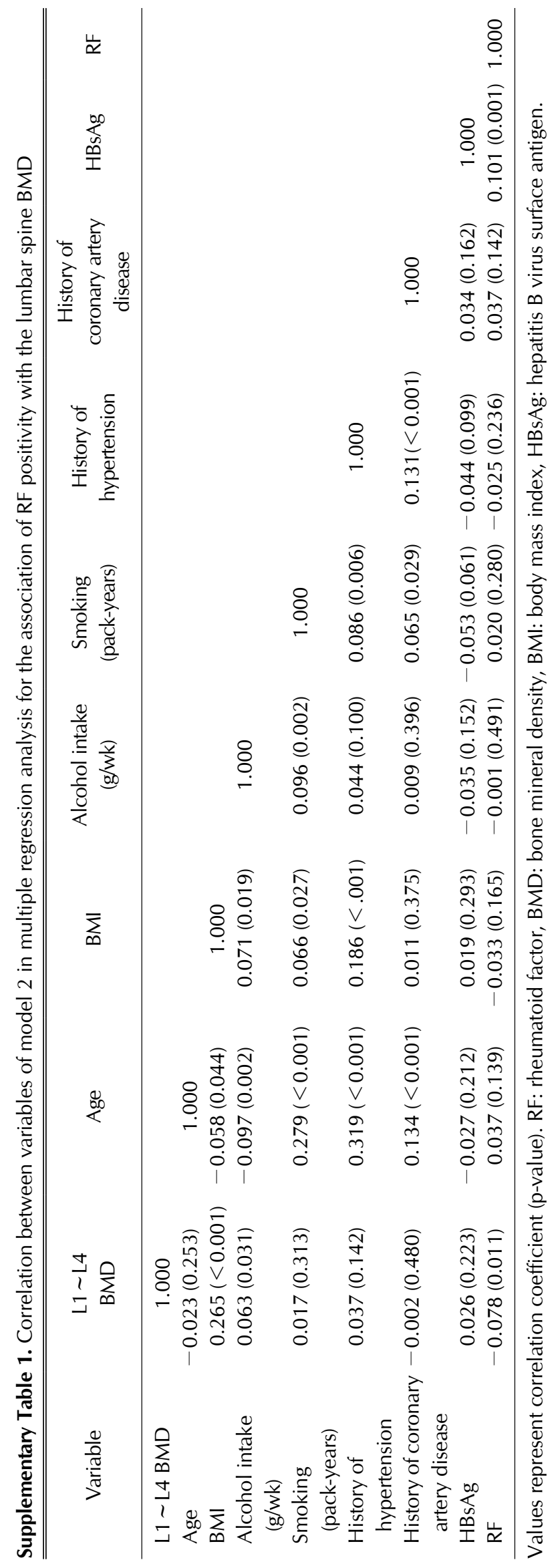

